\title{
Measuring Oxygen Concentration in a Fuel Tank Ullage
}

\author{
William M. Cavage* \\ Federal Aviation Administration, Atlantic City International Airport, NJ 08405 \\ William J. Hughes Technical Center
}

\begin{abstract}
For many years the Federal Aviation Administration (FAA) has been seeking to improve fuel tank safety, particularly center-wing fuel tanks, which were implicated in four fatal explosions over a 10-year period. Fuel tank inerting technologies have been examined by the FAA in depth and evaluated for potential benefit and cost. In support of this, the William J. Hughes Technical Center Fire Safety Branch has examined and evaluated many technologies for measuring oxygen concentration, some of which have been applied for use in a fuel tank ullage. To evaluate existing methods for measuring oxygen concentration in a fuel tank ullage, three different technologies were examined to determine how well they could duplicate calibration gas values, given the method of application, over a range of relevant conditions. Also, each technology/method was installed in a scale fuel tank test article to gauge the ability of the method to accurately determine the oxygen concentration under simulated commercial transport airplane fuel tank ullage conditions. These technologies and methods are 1) a typical lab-based galvanic cell sensor, using a regulated sample train; 2) a light absorption sensor, using an unregulated sample train; 3) an optical fluorescencebased sensor that is used in situ (in place). Preliminary data has illustrated that the light absorption method as well as the optical fluorescence method of sensing can represent calibration gases in a variable environment with some accuracy, but additional work is needed to adequately compare the capability of each method.
\end{abstract}

\section{Introduction}

For several years the Federal Aviation Administration (FAA) has been seeking to improve fuel tank safety in the commercial transport airplane fleet, given the tragic events of TWA Flight 800 in July 1996. Of particular interest were center-wing fuel tanks, which were implicated by the FAA in four fatal explosions over a period of approximately 10 years. To this end, fuel tank inerting technologies have been examined by the FAA in depth and evaluated for potential benefit and cost. In support of fuel tank safety research and development, the William J. Hughes Technical Center Fire Safety Team has examined and evaluated many technologies for measuring oxygen concentration. Some of these have been applied for measuring oxygen concentration in a fuel tank ullage.

Measuring oxygen in a given environment is accomplished by exposing a gas sample to a sensor designed to respond to a changing amount of oxygen in the sample. The various sensing technologies rely on one of several different natural properties of the sensor, as it relates to oxygen, to change the voltage in a circuit, or more directly, the number of counts on a digital encoder, spectrometer, or analog-to-digital converter. Virtually all oxygen sensors do not sense the volume percentage of oxygen in a sample, but rather, the partial pressure of oxygen in a gas sample. The analyzer is then calibrated for volume percent oxygen, given the gas sample conditions. This can be accomplished by a highly regulated gas sample or by compensating for changes in pressure and temperature in the gas sample through a multi-dimensional calibration. It is also possible to use a sensor in situ, or literally in place, thus avoiding the entire gas-sampling process. This requires prior knowledge of how the sensor will react, given all the potential changes in the sensor environment (i.e., pressure, temperature, humidity, etc.), and generally limits the ability to calibrate the sensor routinely.

To evaluate existing methods for measuring oxygen concentration, three different technologies were examined to determine how well they could duplicate calibration gas values, given the method of application, over a range of conditions. Also, each technology and method was installed in a scale fuel tank test article to gauge the ability of the method to accurately determine the oxygen concentration under simulated commercial transport airplane fuel tank ullage conditions. These technologies and methods were 1) a typical lab-based galvanic cell sensor, using a

* Project Manager, Fire Safety Research, William. J. Hughes Technical Center, Senior Member AIAA 
regulated sample train; 2) a light absorption sensor, using an unregulated sample train; and 3) an optical fluorescence-based sensor that is used in situ.

\section{Methods Examined}

\section{Galvanic Cell}

A galvanic cell is a sensor containing a small amount of a liquid solution that reacts with oxygen, which is retained within a permeable membrane. When a sensor membrane is exposed to a gas sample, oxygen enters or leaves the sensor solution to create an equilibrium condition. The solution in these sensors is separated by an anode and cathode. A fixed-current source changes the voltage across the anode and cathode, given a changing amount of oxygen in the sensor solution. The galvanic cell was employed in a highly regulated sample train designed and built by FAA Fire Safety personnel (often referred to as the FAA method) to evaluate different fuel tank inerting methodologies during flight tests [1].

In an attempt to improve the FAA method, given lessons learned from using the system during flight tests, changes were made to the gas sample system described in reference 1. The forward pressure controller on each channel was replaced with an adjustable needle valve and each channel was given its own back pressure controller. Also, snapback flame arrestors were replaced with lighter, cindered metal arrestors and the liquid traps were removed. A lighter, smaller pump was used, and the sensor electrical fault barriers were removed. Additionally, not relevant to reference 1 , the system was repackaged in a smaller, lighter housing, which was designed to be more resistant to impact, as well as being quieter and easier to maintain.

\section{Light Absorption}

The light absorption sensor uses laser diode light absorption to measure oxygen concentration in a gas sample. The laser diode produces light in the visible spectrum at 760 nanometers, which is absorbed by oxygen. To detect the amount of oxygen, a laser beam is focused through the gas sample onto a detector. Oxygen concentration is inversely proportional to the amount of light reaching the detector. The analyzer automatically zeros for every measurement using tunable laser diode (TLD) technology. Proprietary algorithms are used to interpret peak absorption and line width. The sensor is applied by using a high-volume, low-pressure differential pump to continuously draw a gas sample through the analyzer. The four-channel analyzer then interprets each channel sequentially using the single sensor through a complex series of valves [2]. The type of pump used cannot significantly boost the pressure of the gas sample and requires that the gas sample be drawn and redeposited to the same environment.

\section{Optical Fluorescence}

The optical fluorescence sensor uses the principal of fluorescence quenching, i.e., certain properties of light will change with the partial pressure of oxygen in a given environment. These fiber optic sensors detect changing fluorescence properties (intensity, time decay, etc.) of an oxygen-sensitive fluorophore, which is embedded in a probe, when stimulated by a coherent light source. A spectrometer is used to measure the time frequency data of the reflected light from the flourophore that can be used to interpret oxygen concentration, given a known pressure and temperature of the probe tip [3].

\section{Test Equipment and Procedures}

\section{Test Equipment}

To simulate the needed pressure and temperature of flight, the stated test procedures were performed in an environmental chamber at the William J. Hughes Technical Center in New Jersey. The chamber could be operated at constant temperature and pressure to evaluate each measurement method at discrete points or at varying temperature and pressure to compare each method during a simulated commercial transport flight cycle. The inside dimensions of the chamber are 72 by 71 by 93 inches.

To validate the oxygen measurement methods at various static pressures and temperatures, a device was employed to expose each method to different calibration gases simultaneously. This was accomplished with a PVC pipe that had a calibration gas deposit fitting on one end and a series of fittings along its length, attached to different sensors 
or gas sample tubes. The opposite end of the PVC pipe was open to allow for chamber pressure and temperature to equalize in the pipe.

The fuel tank test article was a welded rectangular aluminum tank, roughly 3 by 3 by 2 feet high. It contained a drain at the bottom and a vent on the top with an additional capped port on the top of the tank for refueling, defueling, and checking the fuel quantity. Through experimentation, the fuel tank was determined to hold 128 gallons. From that fuel quantity, any percentage of full tank capacity could be calculated. A clear tube plumbed into the bottom of the tank was used to determine fuel quantity with graduation marks on the side of the tank. Also, a 12- by 8-inch-rectangular hole was installed in the top of the tank, and covered with aluminum foil, to provide pressure relief in the event of a failure, which could cause an ullage deflagration (explosion). A 16- by 16-inch instrumentation panel was installed in the top of the tank in which various bulkhead fittings were installed to allow access for gas sample tubing and thermocouples as well as depositing inert gas and air for test purposes. Twelve Ktype thermocouples were installed in the fuel tank on the bottom, top, and side surfaces as well as in both the fuel and ullage at various locations. A block diagram of the tank and instrumentation is shown in figure 1.

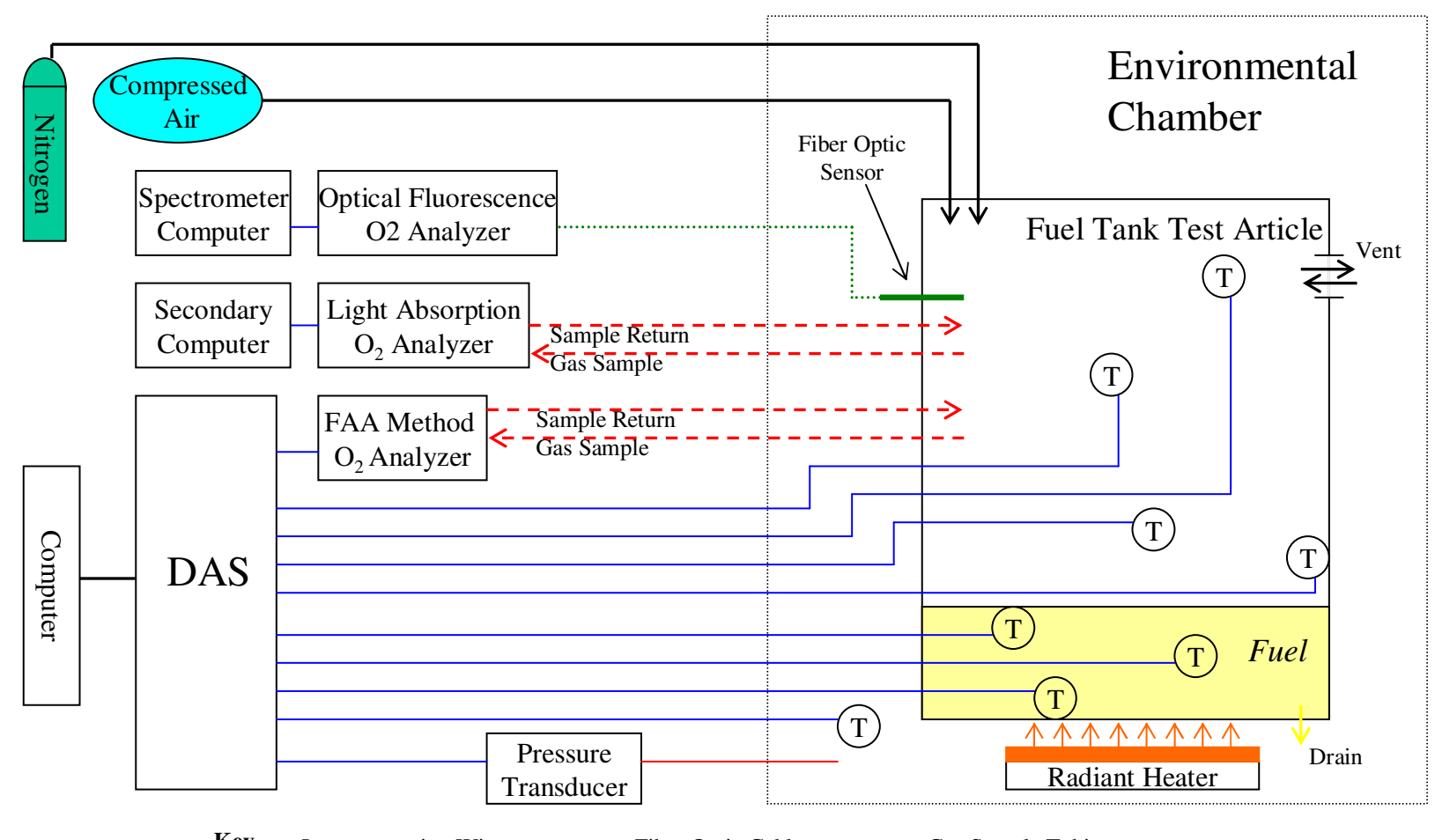

Key Instrumentation Wire - $\quad$ Fiber Optic Cable ……... Gas Sample Tubing - - -

Figure 1. Block Diagram of the Fuel Tank Test Article in the Environmental Chamber with Test Equipment

\section{Test Procedures}

To determine the ability of each oxygen measurement method to duplicate known calibration gases, the PVC tube device was placed in the environment chamber. Two of the analyzers sampled from the PVC tube while the fiber optic sensor was installed in the tube. The chamber was operated at a variety of pressures and temperatures. When conditions in the chamber were stable, the three methods were exposed to three different calibration gases; 14.9-, 10.0-, and 5.00-percent oxygen in a nitrogen balance. The stable reading of each measurement method was recorded for each calibration gas.

To determine the response time of each measurement method, each measurement method was exposed to a stable 5percent oxygen concentration at sea level pressure and lab temperature using calibration gases and the PVC pipe described above. The calibration gas was then switched to 15 percent and the time to measure within 0.1-percent oxygen of a stable reading was recorded. 
To determine how well each oxygen measurement method would be able to track the changing oxygen concentration of a commercial transport airplane fuel tank ullage that was being inerted, each method was installed in the scale fuel tank test article, which contained a small amount of fuel. The test article was then subjected to changes in pressure and temperature typical of commercial transport airplanes while the tank ullage was ventilated with nitrogen at a rate that created oxygen concentration changes expected from a commercial fuel tank inerting system. Figure 2 gives the pressure and temperature profile for the simulated flight with the anticipated ullage oxygen concentration employed

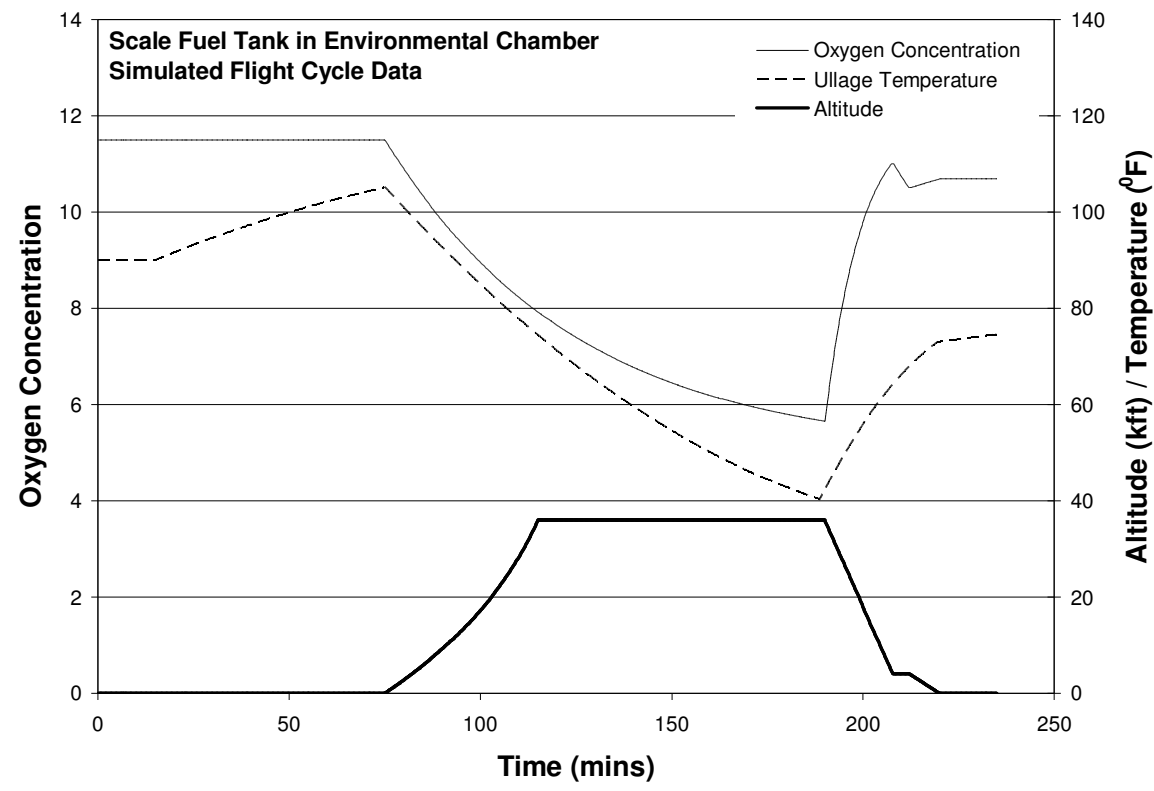

Figure 2. Typical Fuel Tank Ullage Commercial Transport Flight Cycle Conditions

\section{Preliminary Results}

\section{Calibration Gas Results}

Each method was calibrated using a procedure prescribed by the system manufacturer. The FAA method only used fresh air to calibrate. The light absorption method, made by Oxigraf, used two independent calibration gases and a check gas housed with the system rack to calibrate the sensor in the measurement system. The optical fluorescence method, packaged by Aviation Safety Facilitators (ASF), uses a complex multiple-dimensional calibration created at the developer's laboratory and then "aligned" using a single calibration gas, which was used during the experiments, at a single temperature and pressure.

The stable reading from each measurement method and the calculated error at a variety of relevant conditions is given in table 1. The error is calculated as percent difference from the given calibration gas value. These preliminary results indicate that each method can obtain a valid measurement, given the stable conditions identified in the table. It is evident the FAA method duplicated calibration gas values better than the other two methods over the test conditions. The light absorption method could not obtain data during the 3.300 psia absolute conditions. This was attributed to a leak in the systems gas sample train. Also, the optical fluorescence method exhibited large errors at reduced pressures.

The response time examination results are shown in table 2. It was unexpected that the optical fluorescence method, which is in situ, had the worst response time of the three methods examined. This is attributed to the developmental software that analyzes the data, which has not been optimized for speed at this time. The particular set of conditions was chosen because the 5- to 15-percent oxygen chosen relates to the expected full range of conditions expected in a commercial transport fuel tank ullage. The $0.1 \%$ difference is the observed repeatability of this type of measurement. Therefore this number shows how quickly the method can span the expected range within the repeatability of the instrument. 
Table 1. Preliminary Results Comparing Oxygen Measurement Methods Exposed to Calibration Gases

\begin{tabular}{|c|c|c|c|c|c|c|c|}
\hline \multirow[b]{2}{*}{ Condition } & \multirow[b]{2}{*}{ Cal Gas } & \multicolumn{2}{|c|}{ FAA Method } & \multicolumn{2}{|c|}{ Oxigraf } & \multicolumn{2}{|c|}{ ASF } \\
\hline & & Value & Error & Value & Error & Value & Error \\
\hline \multirow[t]{3}{*}{ Sea Level, 75 deg F } & 14.9 & 15.0 & $0.7 \%$ & 15.1 & $1.3 \%$ & 14.8 & $0.7 \%$ \\
\hline & 10.0 & 10.0 & $0.0 \%$ & 10.1 & $1.0 \%$ & 9.9 & $1.0 \%$ \\
\hline & 5.0 & 5.0 & $0.0 \%$ & 5.0 & $0.0 \%$ & 3.9 & $22.0 \%$ \\
\hline \multirow[t]{3}{*}{5.555 psia, 74 deg $F$} & 14.9 & 15.0 & $0.7 \%$ & 15.0 & $0.7 \%$ & 12.1 & $18.8 \%$ \\
\hline & 10.0 & 10.0 & $0.0 \%$ & 10.0 & $0.0 \%$ & 6.1 & $38.8 \%$ \\
\hline & 5.0 & 5.1 & $2.0 \%$ & 5.2 & $4.0 \%$ & 1.4 & $71.1 \%$ \\
\hline \multirow[t]{3}{*}{3.300 psia, 77 deg $F$} & 14.9 & 15.0 & $0.7 \%$ & & & 8.5 & $42.8 \%$ \\
\hline & 10.0 & 10.0 & $0.0 \%$ & & & 3.7 & $62.6 \%$ \\
\hline & 5.0 & 5.1 & $2.0 \%$ & & & 1.1 & $77.4 \%$ \\
\hline
\end{tabular}

Table 2. Preliminary Results Comparing Oxygen Measurement Methods Response Time

\begin{tabular}{|c|c|c|c|}
\hline \multirow[b]{2}{*}{ Condition } & \multicolumn{3}{|c|}{ |Time to Achieve $0.1 \%$ Difference (seconds) } \\
\hline & FAA Method & Oxigraf & ASF \\
\hline $\begin{array}{l}\text { Change Stable 5\% Cal Gas } \\
\text { to } 15 \% \text { Cal Gas }\end{array}$ & 49 & 24 & 55 \\
\hline
\end{tabular}

\section{Future Work}

Due to equipment failure and developmental short comings associated with the different measurement methods, most of the test plan was not completed. The environmental facility is scheduled to be repaired and on-line by the middle of February 2009, when work will resume and hopefully be completed shortly thereafter. This will also allow time for changes needed due to developmental problems.

\section{References}

${ }^{1}$ Burns, Michael and Cavage, William M., "A Description and Analysis of the FAA Onboard Oxygen Analysis System," FAA Report DOT/FAA/AR-TN03/52, June 2003.

${ }^{2}$ Thorson, Eric, "Instruction Manual Oxigraf O2N2 Flight Test Oxygen Analyzer," Rev A5, October 2006, p 6.

${ }^{3}$ Krihak, M., Murtagh, M., and Shahriari, M. R., "Fiber Optic Oxygen Sensors Based on the Sol-Gel Coatings Technique," SPIE Chemical, Biological, and Environmental Fiber Sensors VIII Conference Proceedings, Vol. 2836, pp. 105-115. 